\title{
On Class of Fractional-Order Chaotic or Hyperchaotic Systems in the Context of the Caputo Fractional-Order Derivative
}

\author{
Ndolane Sene $\mathbb{D}^{1}$ and Ameth Ndiaye $\mathbb{D}^{2}$ \\ ${ }^{1}$ Laboratoire Lmdan, Département de Mathématiques de la Décision, Université Cheikh Anta Diop de Dakar, \\ Faculté des Sciences Economiques et Gestion, Dakar Fann BP 5683, Senegal \\ ${ }^{2}$ Département de Mathématiques, Faculté des Sciences et Technologies de L'Education et de la Formation, \\ Université Cheikh Anta Diop de Dakar, BP 5036 Dakar Fann, Senegal \\ Correspondence should be addressed to Ndolane Sene; ndolanesene@yahoo.fr
}

Received 18 September 2020; Revised 11 October 2020; Accepted 18 October 2020; Published 17 November 2020

Academic Editor: Chokkalingam Ravichandran

Copyright (c) 2020 Ndolane Sene and Ameth Ndiaye. This is an open access article distributed under the Creative Commons Attribution License, which permits unrestricted use, distribution, and reproduction in any medium, provided the original work is properly cited.

In this paper, we consider a class of fractional-order systems described by the Caputo derivative. The behaviors of the dynamics of this particular class of fractional-order systems will be proposed and experienced by a numerical scheme to obtain the phase portraits. Before that, we will provide the conditions under which the considered fractional-order system's solution exists and is unique. The fractional-order impact will be analyzed, and the advantages of the fractional-order derivatives in modeling chaotic systems will be discussed. How the parameters of the model influence the considered fractional-order system will be studied using the Lyapunov exponents. The topological changes of the systems and the detection of the chaotic and hyperchaotic behaviors at the assumed initial conditions and the considered fractional-order systems will also be investigated using the Lyapunov exponents. The investigations related to the Lyapunov exponents in the context of the fractional-order derivative will be the main novelty of this paper. The stability analysis of the model's equilibrium points has been focused in terms of the Matignon criterion.

\section{Introduction}

The subject of fractional differential equations has recently evolved as an interesting and popular field of research; see the interesting papers $[1,2]$. In fact, fractional derivatives provide an excellent tool for describing memory and hereditary properties of various materials and processes. More and more researchers have found that fractional differential equations play important roles in many pieces of research areas, such as physics [3-6], fluid dynamics [7], population dynamics [8], biotechnology [8], economics $[1,9]$, and mathematical modeling $[2,7]$. For examples of the recent advancements in the fractional derivatives and their applications, see $[10,11]$. For more investigations, we advise readers to refer to papers [12-15] and the references cited therein. Nowadays, it is established in the literature that the fractional calculus has many advantages in modeling real-world problems due to the memory effect. Another fundamental property of fractional calculus can be observed in physics, notably in diffusion fractional differential equations. There exist specific diffusion types as subdiffusion, hyperdiffusion, and superdiffusion $[5,6]$, which are generated by particular values of the fractional-order derivative and cannot be observed with an integer-order derivative. In other words, new diffusion types appear with fractional operators and fractional calculus.

The Caputo derivative [16-18] is one of the most used fractional derivatives in the literature. But many other fractional derivatives exist too; see $[19,20]$. In this paper, we experienced the Caputo derivative in modeling the fractional-order chaotic and hyperchaotic systems. We notice many types of chaotic systems in the literature: Chen chaotic 
systems [21-23], Lu et al.'s chaotic system [24], Chua's chaotic system [25], new class of chaotic systems [26], Lorenz attractor [12], and many others [27, 28]. Many modifications to propose new chaotic systems exist too; see [29-33]. The chaotic systems have much importance in modeling real-world problems in many fields: in physics $[34,35]$, in economics and finance $[1,27,36]$, and in electrical circuits notably $[37,38]$. Nowadays, there exist many investigations related to chaotic and hyperchaotic systems. Akgul et al. [29] presented a new four-scroll three-dimensional chaotic attractor and applied it in science and engineering. $\mathrm{Xu}$ et al. [39] proposed a new chaotic system with a self-excited attractor; they mainly studied entropy measurement, signal encryption, and parameter estimations. He et al. [40] proposed a numerical analysis of a fractional-order chaotic system by using the conformable fractional-order derivative. In [41], Rajagopal et al. present an investigation related to the chaotic chameleon, the dynamics of the model are analyzed, and the model is applied in modeling the electrical circuits. In the context of fractional-order derivative, Petras proposed classes of the fractional-order chaotic systems in [42]. In [33], Vaidyanathan et al. offered a four-dimensional chaotic hyperjerk approach, studied its synchronization, and gave the implementation of the model in the context of electrical circuits. In [32], Ren et al. proposed an interesting paper addressing new chaotic flow with the hidden attractor. For more investigations on chaotic systems, see [43-46].

Our motivations come from the fact that it is known in the literature that the fractional-order derivative admits the memory effect property, and it generalizes the integer-order derivative to non-integer-order derivatives. The novelty of this paper is that it proposes the phase portraits of the new fractional-order model proposed by Lu et al. in [24]. We will exercise a numerical scheme that uses the numerical discretization of the fractional integral. The main contribution of this paper will be to characterize the existence of chaotic behaviors or hyperchaotic behaviors in the fractional context using the calculations of the Lyapunov exponents. We will also analyze the impact generated by the variations of the parameters of the model in the chaotic or hyperchaotic behaviors using the bifurcation diagrams or Lyapunov exponents as possible. As will be noticed, fractional-order derivatives are useful tools to obtain new types of chaotic or hyperchaotic behaviors. Before the above investigations, we will prove the existence and the uniqueness of the solution of the considered system using Banach-fixed theorem in fractional context. We will close the investigations by investigating the stability analysis of the equilibrium points of the proposed model. We will use the Matignon criterion known for the stability in the fractional context.

We divided the paper to the following parts: In Section 2, we recall the definitions used in our investigations. In Section 3, we describe Lu et. al's fractional-order system considered in this paper. In Section 4, we propose the uniqueness and the existence of the supposed $\mathrm{Lu}$ et. al's fractional-order system. In Section 5, the numerical discretization for the phase portraits of the fractional-order model is presented, and the solutions are depicted graphically. In Section 6, we work on the detection of the chaotic or hyperchaotic behaviors considering the Lyapunov exponents. In Section 7, we investigate the stability using the Matignon criterion used in fractional calculus for the stability analysis. In Section 8, we give all conclusions related to our contribution and provide future directions for researches.

\section{Basic Fractional Calculus Operators}

Let us recall all the definitions and properties that are essential for our investigations. In this paper, we are interested in the Riemann-Liouville integral, the Caputo derivative, and all properties related to these operators $[17,18]$.

Definition 1. The Riemann-Liouville integral of order $\alpha>0$ for a continuous function defined on $f \in C^{n}([0,1], \mathbb{R})$ is given by

$$
I^{\alpha} f(t)=\frac{1}{\Gamma(\alpha)} \int_{0}^{t}(t-\tau)^{\alpha-1} f(\tau) \mathrm{d} \tau,
$$

with $\Gamma(\alpha):=\int_{0}^{\infty} e^{-u} u^{\alpha-1} \mathrm{~d} u$.

Definition 2. If $f \in C^{n}([0,1], \mathbb{R})$ and $n-1<\alpha \leq n$, then the Caputo fractional derivative is given by

$$
\begin{aligned}
D_{c}^{\alpha} f(t) & =I^{n-\alpha} \frac{\mathrm{d}^{n}}{\mathrm{~d} t^{n}}(f(t)) \\
& =\frac{1}{\Gamma(n-\alpha)} \int_{0}^{t}(t-s)^{n-\alpha-1} f^{(n)}(s) \mathrm{d} s .
\end{aligned}
$$

Lemma 1. Taking $n \in \mathbb{N}^{*}$ and $n-1<\alpha<n$, the general solution of $D_{c}^{\alpha} u(t)=0$ is obtained as follows:

$$
u(t)=\sum_{i=0}^{n-1} c_{i} t^{i},
$$

such that $c_{i} \in \mathbb{R}, i=0,1,2, \ldots, n-1$.

Lemma 2. Taking $n \in \mathbb{N}^{*}$ and $n-1<\alpha<n$, we have

$$
I^{\alpha} D_{c}^{\alpha} u(t)=u(t)+\sum_{i=0}^{n-1} c_{i} t^{i},
$$

such that $c_{i} \in \mathbb{R}, i=0,1,2, \ldots, n-1$.

The following result establishes a relation between the integral representation and the differential system given in (13)-(15).

Lemma 3. Let $G \in C([0,1])$. Then, we can state that the problem

$$
\left\{\begin{array}{l}
D_{c}^{\alpha} u(t)=G(t), \quad t \in[0,1] \\
u(0)=u_{0} \\
0<\alpha<1
\end{array}\right.
$$

admits the following representation as integral solution: 


$$
u(t)=u_{0}+\frac{1}{\Gamma(\alpha)} \int_{0}^{t}(t-s)^{\alpha-1} G(s) \mathrm{d} s .
$$

Proof. Using Lemma 2, we have

$$
u(t)=u_{0}+\frac{1}{\Gamma(\alpha)} \int_{0}^{t}(t-s)^{\alpha-1} G(s) \mathrm{d} s,
$$

and with the initial condition we have the result.

Let us now transform the above problem to a fixed-point one.

We begin by considering the Banach space,

$$
X:=\{x \in C(J, \mathbb{R})\}, \quad J=[0,1],
$$

and its norm,

$$
\|x\|_{X}=\operatorname{Sup}_{t \in J}|x(t)| .
$$

Then, we consider the operators $T_{1}, T_{2}, T_{3}: X \longrightarrow X$ defined by

$$
\begin{gathered}
T_{1} x(t)=x_{0}+\frac{1}{\Gamma(\alpha)} \int_{0}^{t}(t-s)^{\alpha-1} \\
\cdot\left(-\frac{a b}{a+b} x(s)-y(s) z(s)+c\right) \mathrm{d} s \\
T_{2} y(t)=y_{0}+\frac{1}{\Gamma(\alpha)} \int_{0}^{t}(t-s)^{\alpha-1}(a y(s)+x(s) z(s)) \mathrm{d} s, \\
T_{3} z(t)=z_{0}+\frac{1}{\Gamma(\alpha)} \int_{0}^{t}(t-s)^{\alpha-1}(b z(s)+x(s) y(s)) \mathrm{d} s
\end{gathered}
$$

\section{Lu et. al's Fractional-Order System}

There exist nowadays many chaotic and hyperchaotic systems, as recalled in Introduction. Due to the rapid development of the fractional calculus [47], the existence of the new fractional operators to model real-world problems, and the fact that the fractional operators take into account the memory effect in modeling physical issues, the question related to revisiting the chaotic and hyperchaotic systems in terms of the fractional operators is our motivation. The main problem is how the chaotic and hyperchaotic behaviors can be characterized in the context of the fractional operators. How the Lyapunov exponents under time series evolution and the variations of the parameters of the model can be calculated? How the bifurcation can be interpreted in a fractional context? Many questions appear for modeling chaotic and hyperchaotic systems with fractional operators. In this paper, we will try to answer some of the above questions. In this paper, we consider a class of systems proposed in [24], but here we write it by considering the Caputo fractional derivative. We have the following representation for this type of $\mathrm{Lu}$ et al.'s system:

$$
\begin{aligned}
& D_{c}^{\alpha} x=-\frac{a b}{a+b} x-y z+c, \\
& D_{c}^{\alpha} y=a y+x z, \\
& D_{c}^{\alpha} z=b z+x y .
\end{aligned}
$$

We make the following assumptions related to the initial conditions:

$$
\begin{aligned}
& x(0)=x_{0}, \\
& y(0)=y_{0}, \\
& z(0)=z_{0} .
\end{aligned}
$$

where $0<\alpha<1$ and $a, b, c$ are constants. For the present model, the values of the parameters are as follows: $a=-10$, $b=-4$, and $c=0$. Our new objective is to explain concretely what the influence generated by the fractional-order derivative in modeling the above fractional differential systems is. It will be noticed in the forthcoming section that the local stability of the model depends strongly on the value assigned to the fractional-order derivative. Lu et al.'s fractional model presented in this section has many applications; for example, it can be implemented for electrical circuits. To model electrical circuits using equations (13)-(15), for example, the parameters $a, b$ can denote the intensities of the capacitors $C_{1}$ and $C_{2}$, respectively, and $c$ is an exogenous input, or op-amp, or denotes the intensity of the inductor $L$ or denotes the intensity of the resistor $R$. Furthermore, for example, depending on the considered electrical circuit which we want to implement, $x$ can describe the voltage across the capacitor $C_{1}, y$ can represent the voltage across the capacitor $C_{2}$, and $z$ can represent the electric current into the inductor $L$ or others. Here we give a possible example of implementation to prove that $\mathrm{Lu}$ et al.'s fractional-order model can be used in real-world modeling problems. The mathematical aspects of equations (13)-(15) are under consideration in this paper.

\section{Existence and Uniqueness of the Solutions of the Model}

Now, we show the existence and the uniqueness of the solutions of the system in (13)-(15) by using the Banach fixed-point theorem for contraction mappings.

The Banach fixed-point theorem is recalled in the following theorem.

Theorem 1 (see [48]). Let $\Omega$ be a nonempty closed subset of a Banach space $X$. Then, for any contraction mapping $T$ of $\Omega$ into itself has a unique fixed point.

To prove the existence and uniqueness of our model's solution, we continue with the following theorem.

Theorem 2. Assume that $x, y, z$ are continuous functions satisfying the following condition:

(H1) There exist constants $m_{1}, m_{2}, m_{3}>0$ such that $|x(t)|<m_{1},|y(t)|<m_{2},|z(t)|<m_{3}$.

If $(a b / a+b)<\Gamma(\alpha+1), a<\Gamma(\alpha+1)$, and $b<\Gamma(\alpha+1)$ then the problem in (13)-(15) has a unique solution $x(t)$, 
$y(t) z(t)$ in $X$

Proof. We first show that the operator $T_{1}$ defined by (10) is well defined; that is, we show that $T_{1} \mathscr{B}_{r} \subset \mathscr{B}_{r}$, where

$$
\mathscr{B}_{r}=\{u \in X,\|u\| \leq r\},
$$

with $r>\left(\left(m_{2} m_{3}+c+\left|x_{0}\right| \Gamma(\alpha+1)\right)(a+b)\right) /((a+b) \Gamma(\alpha+1)-$ $a b))$. For any $x \in \mathscr{B}_{r}$ we obtain

$$
\begin{aligned}
\left|T_{1} x(t)\right| \leq & \left|x_{0}\right|+\sup _{t \in J}\left\{\frac{1}{\Gamma(\alpha)} \int_{0}^{t}(t-s)^{\alpha-1}\right. \\
& \left.\cdot\left(\frac{a b}{a+b}|x(s)|+|y(s)||z(s)|+c\right) \mathrm{d} s\right\} \\
\leq & \left|x_{0}\right|+\frac{1}{\Gamma(\alpha)} \int_{0}^{1}(1-s)^{\alpha-1}\left(\frac{a b}{a+b} r+m_{2} m_{3}+c\right) \mathrm{d} s \\
\leq & \left|x_{0}\right|+\frac{(a b / a+b) r+m_{2} m_{3}+c}{\Gamma(\alpha+1)}<r,
\end{aligned}
$$

which implies that $T_{1} x(t) \in \mathscr{B}_{r}$. Moreover, by (10), we obtain $D_{c}^{\alpha} T_{1} x(t)=D_{c}^{\alpha} I^{\alpha}(-(a b /(a+b)) x(t)-y(t) z(t)+c)$ $=-(a b /(a+b)) x(t)-y(t) z(t)+c$. Since $-(a b /(a+b)) x$ $(t)-y(t) z(t)+c$ is continuous on $J, D_{c}^{\alpha} T_{1} x(t)$ is continuous on $J$; that is, $T_{1} \mathscr{B}_{r} \subset \mathscr{B}_{r}$.

Next, we apply the Banach fixed-point theorem to prove that $T_{1}$ has a fixed point. Indeed, just show that $T_{1}$ is contraction map. Let $x_{1}, x_{2} \in X$ and, for $t \in J$, we have

$$
\begin{aligned}
\left\|T_{1} x_{1}-T_{1} x_{2}\right\|_{X} \leq & \sup _{t \in J}\left\{\frac{1}{\Gamma(\alpha)} \int_{0}^{t}(t-s)^{\alpha-1}\right. \\
& \left.\cdot \frac{a b}{a+b}\left|x_{1}(t)-x_{2}(t)\right| \mathrm{d} s\right\} \\
\leq & \frac{a b}{(a+b) \Gamma(\alpha)} \int_{0}^{1}(1-s)^{\alpha-1}\left\|x_{1}-x_{2}\right\|_{X} \mathrm{~d} s \\
\leq & \frac{a b}{(a+b) \Gamma(\alpha+1)}\left\|x_{1}-x_{2}\right\|_{X} .
\end{aligned}
$$

Thus, let $k=(a b /((a+b) \Gamma(\alpha+1)))$; then we have the following relationship:

$$
\left\|T_{1} x_{1}-T_{1} x_{2}\right\|_{X} \leq k\left\|x_{1}-x_{2}\right\|_{X} .
$$

Then, under the assumption that $k<1$, it is shown that $T_{1}$ is a contraction mapping.

With the same method, we show that $T_{2}$ is well defined and is a contraction mapping. We will show that $T_{2} \mathscr{B}_{r}^{\prime} \subset \mathscr{B}_{r}^{\prime}$, where

$$
\mathscr{B}_{r}^{\prime}=\left\{u \in X,\|u\| \leq r^{\prime}\right\}
$$

with $r^{\prime}>\left(\left(m_{1} m_{3}+\left|y_{0}\right| \Gamma(\alpha+1)\right) /(\Gamma(\alpha+1)-a)\right)$. For any $y \in \mathscr{B}_{r}^{\prime}$, we obtain

$$
\begin{aligned}
\left|T_{2} y(t)\right| \leq & \left|y_{0}\right|+\sup _{t \in J}\left\{\frac{1}{\Gamma(\alpha)} \int_{0}^{t}(t-s)^{\alpha-1}(a|y(s)|\right. \\
& +|x(s)||z(s)|) \mathrm{d} s\} \\
\leq & \left|y_{0}\right|+\frac{1}{\Gamma(\alpha)} \int_{0}^{1}(1-s)^{\alpha-1}\left(a r^{\prime}+m_{1} m_{3}\right) \mathrm{d} s \\
\leq & \left|y_{0}\right|+\frac{m_{1} m_{3}+a r^{\prime}}{\Gamma(\alpha+1)}<r^{\prime},
\end{aligned}
$$

which implies that $T_{2} y(t) \in \mathscr{B}_{r}^{\prime}$. With the same arguments, we have $T_{2} \mathscr{B}_{r}^{\prime} \subset \mathscr{B}_{r}^{\prime}$. For the contraction of $T_{2}$, let $y_{1}, y_{2} \in X$ and, for any $t \in J$, we have

$$
\begin{aligned}
\left\|T_{2} y_{1}-T_{2} y_{2}\right\|_{X} & \leq \sup _{t \in J}\left\{\frac{1}{\Gamma(\alpha)} \int_{0}^{t}(t-s)^{\alpha-1} a\left|y_{1}(t)-y_{2}(t)\right| \mathrm{d} s\right\} \\
& \leq \frac{a}{\Gamma(\alpha)} \int_{0}^{1}(1-s)^{\alpha-1}\left\|y_{1}-y_{2}\right\|_{X} \mathrm{~d} s \\
& \leq \frac{a}{\Gamma(\alpha+1)}\left\|y_{1}-y_{2}\right\|_{X} .
\end{aligned}
$$

The inequality $a<\Gamma(\alpha+1)$ shows that $T_{2}$ is contraction mapping.

As a consequence of the Banach fixed-point theorem, the system in (13)-(15) has unique solution $x(t), y(t), z(t) \in X$.

Now we will show with the method that $T_{3}$ is well defined and is a contraction mapping. Let us consider the ball

$$
\mathscr{B}_{r}^{\prime \prime}=\left\{u \in X,\|u\| \leq r^{\prime \prime}\right\},
$$

where $r^{\prime \prime}>\left(\left(m_{1} m_{2}+\left|z_{0}\right| \Gamma(\alpha+1)\right) /(\Gamma(\alpha+1)-b)\right)$. For any $z \in \mathscr{B}_{r}^{\prime \prime}$, we obtain

$$
\begin{aligned}
\left|T_{3} z(t)\right| \leq & \left|z_{0}\right|+\sup _{t \in J}\left\{\frac{1}{\Gamma(\alpha)} \int_{0}^{t}(t-s)^{\alpha-1}\right. \\
& \cdot(|x(s)||(s)|+b|z(s)|) \mathrm{d} s\} \\
\leq & \left|z_{0}\right|+\frac{1}{\Gamma(\alpha)} \int_{0}^{1}(1-s)^{\alpha-1}\left(m_{1} m_{2}+b r^{\prime \prime}\right) \mathrm{d} s \\
\leq & \left|z_{0}\right|+\frac{m_{1} m_{2}+b r^{\prime \prime}}{\Gamma(\alpha+1)}<r^{\prime \prime},
\end{aligned}
$$

which implies that $T_{3} y(t) \in \mathscr{B}_{r}^{\prime \prime}$. Moreover, by the same argument introduced above, we have $D_{c}^{\alpha} T_{3} z(t)=x(t)$ $y(t)+b z(t)$, which is continuous. That is, $T_{3} B_{r}^{\prime \prime} \subset B_{r}^{\prime \prime}$. Indeed, it is enough to show that $T_{3}$ is a contraction map. Let $z_{1}, z_{2} \in X$ and, for any $t \in J$, we have 


$$
\begin{aligned}
\left\|T_{3} z_{1}-T_{3} z_{2}\right\|_{X} & \leq \sup _{t \in J}\left\{\frac{1}{\Gamma(\alpha)} \int_{0}^{t}(t-s)^{\alpha-1} b\left|z_{1}(t)-z_{2}(t)\right| \mathrm{d} s\right\} \\
& \leq \frac{b}{\Gamma(\alpha)} \int_{0}^{1}(1-s)^{\alpha-1}\left\|z_{1}-z_{2}\right\|_{X} \mathrm{~d} s \\
& \leq \frac{b}{\Gamma(\alpha+1)}\left\|z_{1}-z_{2}\right\|_{X} .
\end{aligned}
$$

The inequality $b<\Gamma(\alpha+1)$ shows that $T_{3}$ is contraction mapping.

Then we have that $T_{1}, T_{2}, T_{3}$ are now well defined and are contraction mappings. As a consequence of the Banach fixed-point theorem, we get the result. This ends the proof.

The existence and uniqueness of the model's solution will be beneficial because it will justify the used numerical scheme's stability in this present work. Fixed points are, therefore, of paramount importance in many areas of mathematics, sciences, and engineering; for more interesting articles related to the pertinence of the fixed-point theory, the following references are useful and interesting: $[14,47,49]$.

\section{Approximations for the Fractional- Order System}

This section is devoted to presenting the numerical scheme, which we will apply to get the phase portraits and the approximate solutions of our fractional-order system. It is well known that there exist many methods to obtain the solutions of the fractional differential equations, like the analytical methods and the numerical methods. In the context of fractional chaotic or hyperchaotic systems, the use of analytical techniques is not possible in many contexts due to the nonlinearities of the systems' drift functions. Alternatively, to this inconvenience, the numerical scheme is proposed. The advantages of the numerical methods are that they give more perfect approximate solutions of the fractional-order differential equations. The implementation of the numerical scheme proposed in this section uses the Riemann-Liouville fractional integral. The numerical discretization of the Riemann-Liouville derivative is well known in the literature [50]. The numerical scheme is not complicated to be implemented, and the approximate solutions converge as well to the exact solutions; furthermore, the numerical scheme is stable. After the obtention of the approximations of our model, we will depict the solutions with different values of the fractional-order derivative. That will permit us to see the impact of the fractional-order derivative in the new $\mathrm{Lu}$ et al.'s model with fractionalorder derivative. The numerical scheme that we apply in this section uses the discretization of the Riemann-Liouville integral. Let the following functions be obtained with the fractional model in (13)-(15):

$$
\begin{aligned}
& E(t, x)=-\frac{a b}{a+b} x-y z+c, \\
& F(t, y)=a y+x z, \\
& G(t, z)=b z+x y .
\end{aligned}
$$

The solution of Lu et al.'s fractional model in (13)-(15) is given by the following equations:

$$
\begin{aligned}
& x(t)=x(0)+I^{\alpha} E(t, x), \\
& y(t)=y(0)+I^{\alpha} F(t, y), \\
& z(t)=z(0)+I^{\alpha} G(t, z) .
\end{aligned}
$$

Considering discrete time $t_{n}$ and applying it in the previous equations, we have the following relationships:

$$
\begin{aligned}
& x\left(t_{n}\right)=x(0)+I^{\alpha} E\left(t_{n}, x\right), \\
& y\left(t_{n}\right)=y(0)+I^{\alpha} F\left(t_{n}, y\right), \\
& z\left(t_{n}\right)=z(0)+I^{\alpha} G\left(t_{n}, z\right) .
\end{aligned}
$$

We now choose the step size $h$, and we define the time $t_{n}$ as $t_{n}=n h$; furthermore, considering the classical discretization of the fractional integral, the integral parts of equations (29)-(31) can be represented as follows:

$$
\begin{aligned}
& I^{\alpha} E\left(t_{n}, x\right)=h^{\alpha} \sum_{j=1}^{n} \delta_{n-j} E\left(t_{j}, z_{j}\right), \\
& I^{\alpha} F\left(t_{n}, y\right)=h^{\alpha} \sum_{j=1}^{n} \delta_{n-j} F\left(t_{j}, z_{j}\right), \\
& I^{\alpha} G\left(t_{n}, z\right)=h^{\alpha} \sum_{j=1}^{n} \delta_{n-j} G\left(t_{j}, z_{j}\right),
\end{aligned}
$$

with

$$
\delta_{n}^{(\alpha)}=\frac{(n-1)^{\alpha}-n^{\alpha}(n-\alpha-1)}{\Gamma(2+\alpha)} .
$$

Taking into account equations (32)-(34) and the parameters of the discretization, the numerical scheme that we will use to depict the portraits of Lu et al.'s fractional-order model in (13)-(15) is represented as follows:

$$
\begin{aligned}
& x\left(t_{n}\right)=x(0)+h^{\alpha}\left[\bar{\delta}_{n}^{(\alpha)} E(0)+\sum_{j=1}^{n} \delta_{n-j}^{(\alpha)} E\left(t_{j}, x_{j}\right)\right], \\
& y\left(t_{n}\right)=y(0)+h^{\alpha}\left[\bar{\delta}_{n}^{(\alpha)} F(0)+\sum_{j=1}^{n} \delta_{n-j}^{(\alpha)} F\left(t_{j}, y_{j}\right)\right], \\
& z\left(t_{n}\right)=z(0)+h^{\alpha}\left[\bar{\delta}_{n}^{(\alpha)} G(0)+\sum_{j=1}^{n} \delta_{n-j}^{(\alpha)} G\left(t_{j}, z_{j}\right)\right],
\end{aligned}
$$

where, for $n=1,2, \ldots$, the parameter $\delta$ is expressed in the following form: 


$$
\begin{aligned}
& \delta_{0}^{(\alpha)}=\frac{1}{\Gamma(2+\alpha)}, \\
& \delta_{n}^{(\alpha)}=\frac{(n-1)^{\alpha+1}-2 n^{\alpha+1}+(n+1)^{\alpha+1}}{\Gamma(2+\alpha)},
\end{aligned}
$$

and the discretizations of the functions are

$$
\begin{aligned}
& E\left(t_{j}, x_{j}\right)=-\frac{a b}{a+b} x_{j}-y_{j} z_{j}+c, \\
& F\left(t_{j}, y_{j}\right)=a y_{j}+x_{j} z_{j}, \\
& G\left(t_{j}, z_{j}\right)=b z_{j}+x_{j} y_{j} .
\end{aligned}
$$

We suppose that $x\left(t_{n}\right), y\left(t_{n}\right)$, and $z\left(t_{n}\right)$ are the approximate solutions of $\mathrm{Lu}$ et al.'s fractional model in (13)-(15) and $x_{n}, y_{n}$, and $z_{n}$ are the exact solutions of equations (13)-(15); then the residual functions can classically be expressed as follows:

$$
\begin{aligned}
\left|x\left(t_{n}\right)-x_{n}\right| & =\mathcal{O}\left(h^{\min \{\alpha+1,2\}}\right), \\
\left|y\left(t_{n}\right)-y_{n}\right| & =\mathcal{O}\left(h^{\min \{\alpha+1,2\}}\right), \\
\left|z\left(t_{n}\right)-z_{n}\right| & =\mathcal{O}\left(h^{\min \{\alpha+1,2\}}\right) .
\end{aligned}
$$

From those, the convergence of the numerical approximation is obtained when $h$ converges to 0 . The advantage of the presented numerical schemes concerns the stability stage. Note that, from existence and the uniqueness we have, particularly, the Lipschitz continuity of the functions $E, F$, and $G$, which implies the stability of our numerical scheme.

For the illustrations of the previous numerical scheme, we consider three cases to see how the fractional derivative impacts the dynamics of the considered Lu et al.'s fractionalorder system in (13)-(15). The detection of the chaos with the considered fractional-order derivative will be analyzed later by the Lyapunov exponents. These concepts are classic to characterize the detection of chaos. In our first case, we consider the order $\alpha=0.92$ and the values of the parameters of the model for the rest of this section are fixed as $a=-10$, $b=-4$, and $c=0$.

We depict in Figure 1 the dynamics of Lu et al.'s fractional system in (13)-(15) in context of $x, y$, and $z$ directions, with the order $\alpha=0.92$.

We depict in Figure 2 the dynamics of Lu et al.'s fractional-order system in (13)-(15) in context of $y$ and $z$ directions, with the order $\alpha=0.92$.

We depict in Figure 3 the dynamics of Lu et al.'s fractional-order system in (13)-(15) in context of $x$ and $z$ directions, with the order $\alpha=0.92$.

We depict in Figure 4 the dynamics of Lu et al.'s fractional-order system in (13)-(15) in context of $x$ and $y$ directions, with the order $\alpha=0.92$.

In the second case, we increase the value of the order to $\alpha=0.95$, and the values of the parameters of the model do not change and are $a=-10, b=-4$, and $c=0$.

We depict in Figure 5 the dynamics of the considered $\mathrm{Lu}$ et al.'s fractional-order system in (13)-(15) in context of $x, y$, and $z$ directions, with the order $\alpha=0.95$.

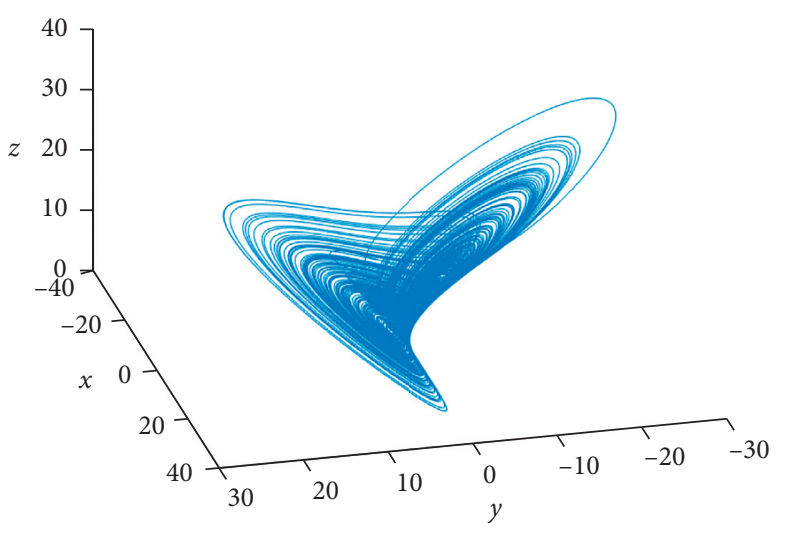

Figure 1: Dynamics of $\mathrm{Lu}$ et al.'s fractional-order system in (13)-(15) with the order $\alpha=0.92$, in context of $x, y$, and $z$ directions.

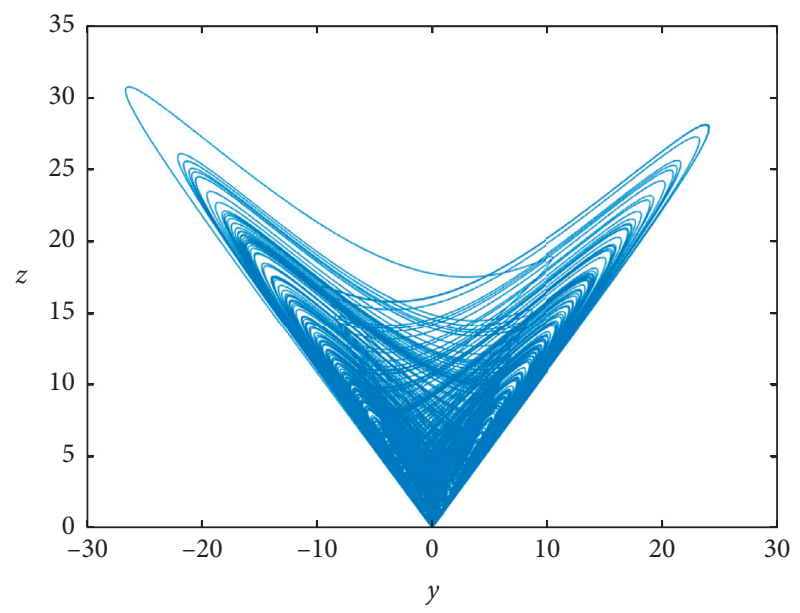

Figure 2: Dynamics of $\mathrm{Lu}$ et al.'s fractional-order system in (13)-(15) with the order $\alpha=0.92$, in context of $y$ and $z$ directions.

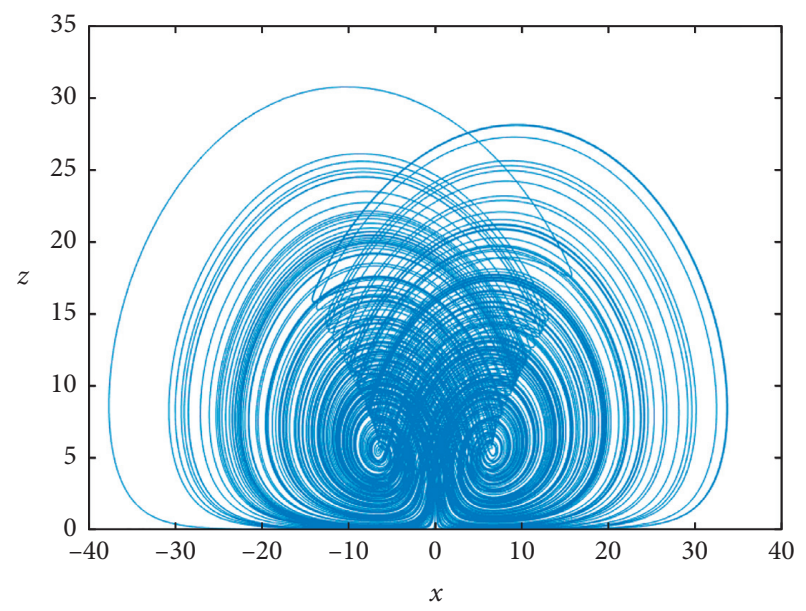

Figure 3: Dynamics of $\mathrm{Lu}$ et al.'s fractional-order system in (13)-(15) with the order $\kappa=0.92$, in context of $x$ and $z$ directions.

We depict in Figure 6 the dynamics of Lu et al.'s fractional-order system in (13)-(15) in context of $y$ and $z$ directions, with the order $\alpha=0.95$. 


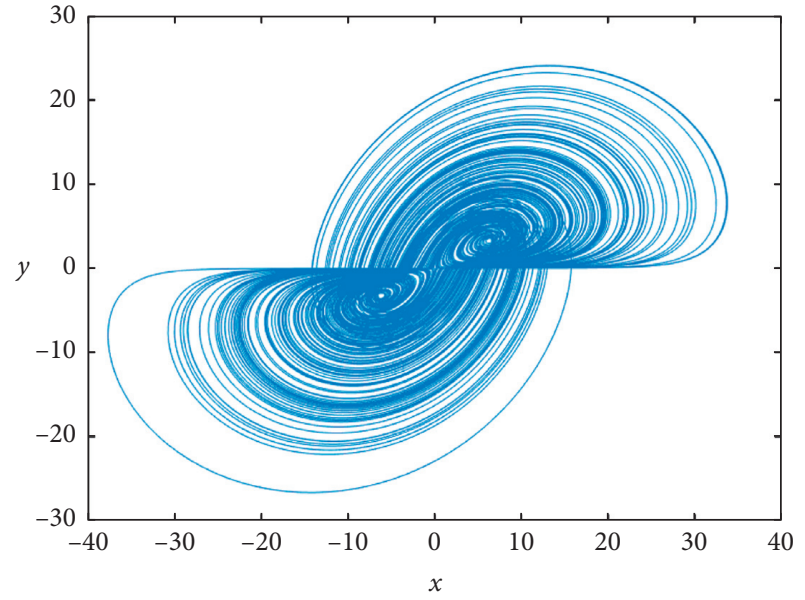

Figure 4: Dynamics of $\mathrm{Lu}$ et al.'s fractional-order system in (13)-(15) with the order $\alpha=0.92$, in context of $x$ and $y$ directions.

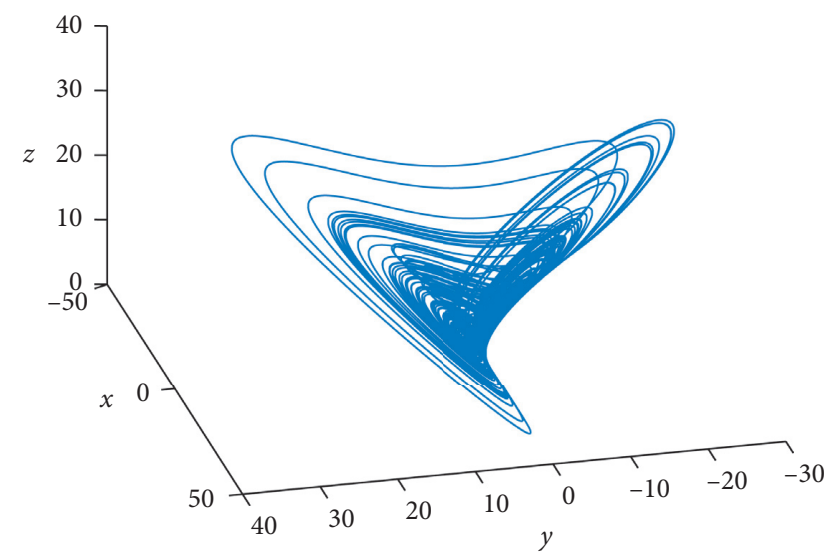

Figure 5: Dynamics of Lu et al.'s fractional-order system with the order $\alpha=0.95$, in context of $x, y$, and $z$ directions.

We depict in Figure 7 the dynamics of Lu et al.'s fractional-order system in (13)-(15) in context of $x$ and $z$ directions, with the order $\alpha=0.95$.

We depict in Figure 8 the dynamics of Lu et al.'s fractional-order system in (13)-(15) in context of $x$ and $y$ directions, with the order $\alpha=0.95$.

Let us now observe what happens when the order is $\alpha=0.88$. We conserve the values of the parameters of the model as $a=-10, b=-4$, and $c=0$.

We depict in Figure 9 the dynamics of Lu et al.'s fractional-order system in (13)-(15) in context of $x, y$, and $z$ directions, with the order $\alpha=0.88$.

We depict in Figure 10 the dynamics of $\mathrm{Lu}$ et al.'s fractional-order system in (13)-(15) in context of $y$ and $z$ directions, with the order $\alpha=0.88$.

We depict in Figure 11 the dynamics of $\mathrm{Lu}$ et al.'s fractional-order system in (13)-(15) in context of $x$ and $z$ directions, with the order $\alpha=0.88$.

We depict in Figure 12 the dynamics of $\mathrm{Lu}$ et al.'s fractional-order system in (13)-(15) in context of $x$ and $y$ directions, with the order $\alpha=0.88$.

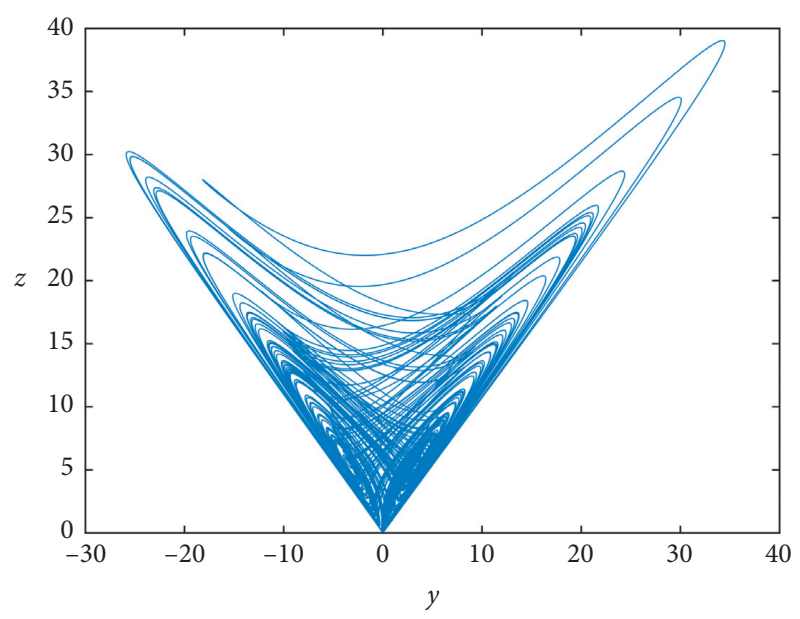

FIgURE 6: Dynamics of Lu et al.'s fractional-order system with the order $\alpha=0.95$, in context of $y$ and $z$ directions.

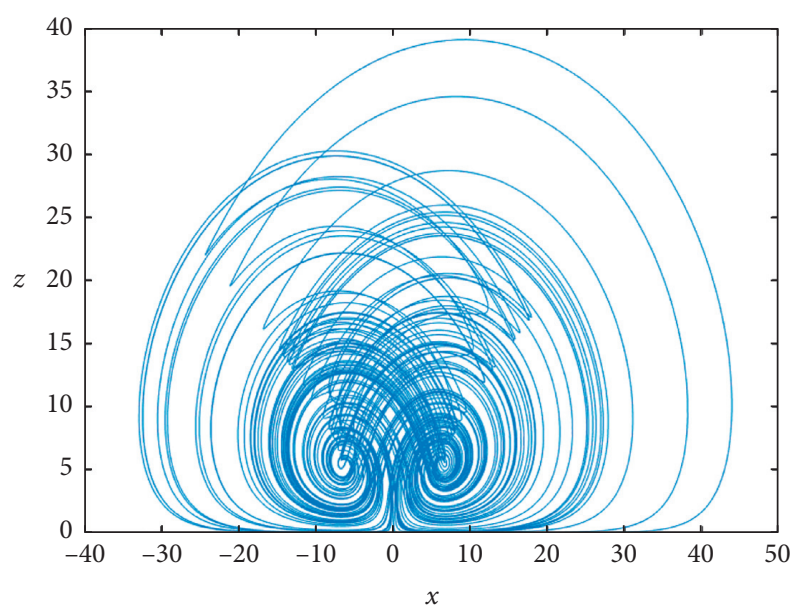

Figure 7: Dynamics of Lu et al.'s fractional-order system with the order $\alpha=0.95$, in context of $x$ and $z$ directions.

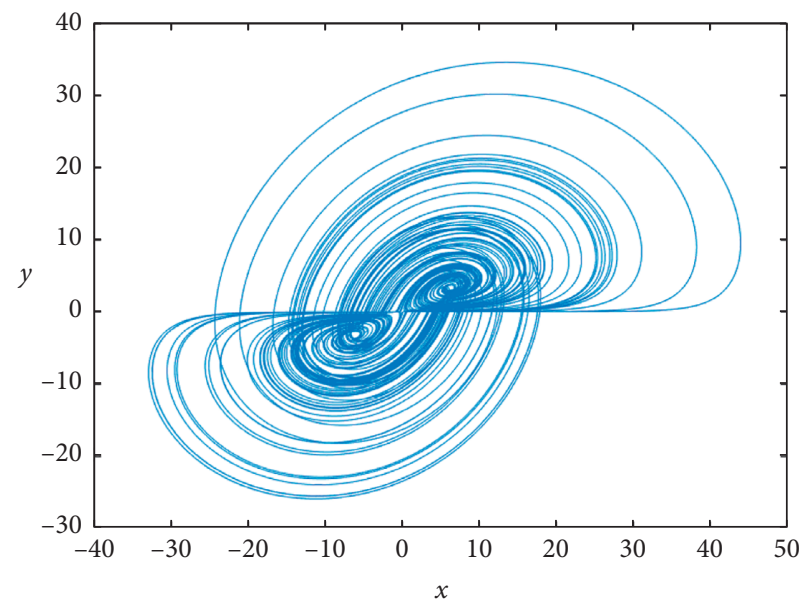

Figure 8: Dynamics of $\mathrm{Lu}$ et al.'s fractional-order system in (13)-(15) with the order $\alpha=0.95$, in context of $x$ and $y$ directions. 


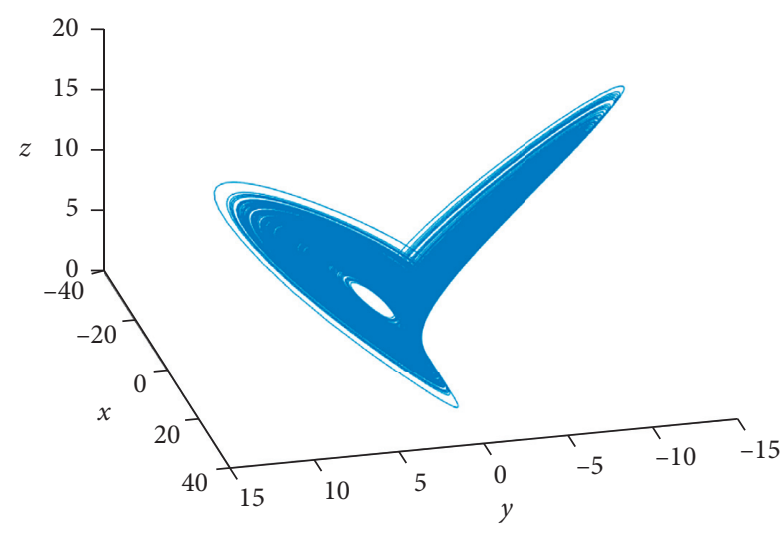

FIGURE 9: Dynamics of $\mathrm{Lu}$ et al.'s fractional-order system in (13)-(15) with the order $\alpha=0.88$, in context of $x, y$, and $z$ directions.

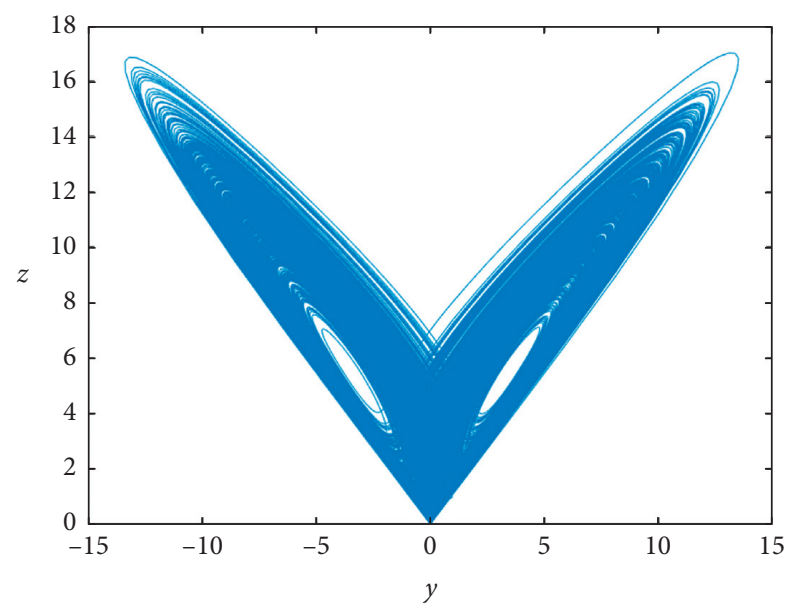

FIGURE 10: Dynamics of Lu et al.'s fractional-order system in (13)-(15) with the order $\alpha=0.88$, in context of $y$ and $z$ directions.

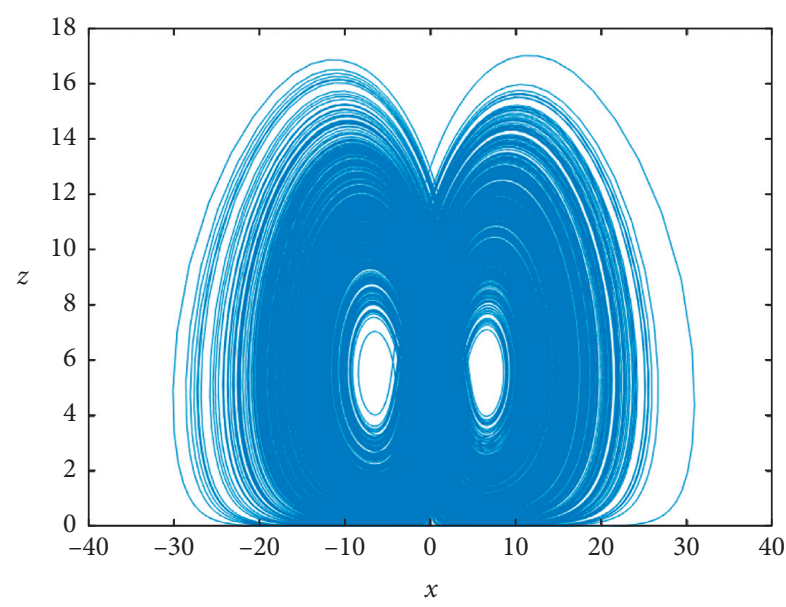

Figure 11: Dynamics of $\mathrm{Lu}$ et al.'s fractional-order system in (13)-(15) with the order $\alpha=0.88$, in context of $x$ and $z$ directions.

In conclusion, we observe that the fractional-order derivative can significantly influence the dynamics of $\mathrm{Lu}$ et al.'s fractional-order system in (13)-(15). How we can explain the

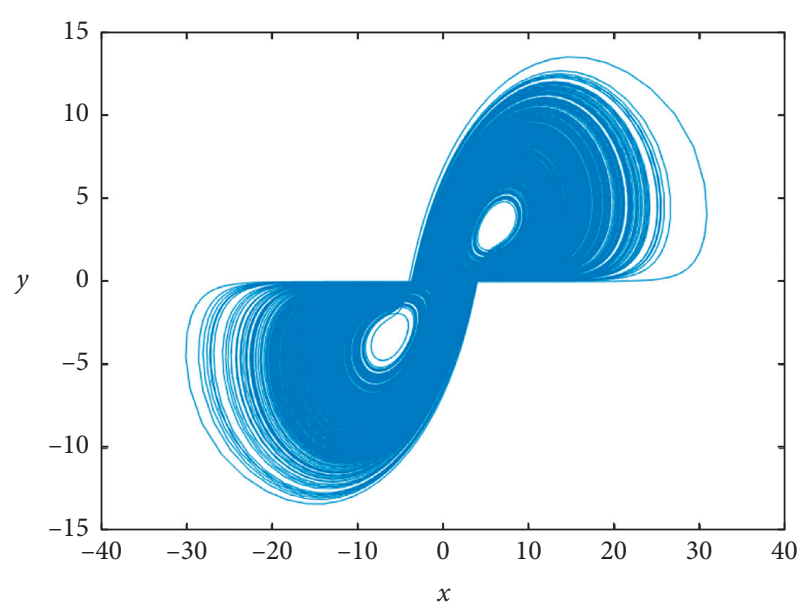

Figure 12: Dynamics of Lu et al.'s fractional-order system with the order $\alpha=0.88$, in context of $x$ and $y$ directions.

variations in the dynamics when the fractional-order derivative varies will be focused on in the next sections. Furthermore, there exist interesting methods described in [51], which can help in future investigations and solution methodologies.

\section{Characterization of the Chaos Behaviors}

In this section, we study the impact of the variations of the model's parameters using the Lyapunov exponents. We also explain the variations of the dynamics observed in the previous section when the fractional derivative operator's order varies in time. Before that, we construct the Jacobian matrix necessary for the calculations of the Lyapunov exponents. We get the following Jacobian matrix:

$$
J=\left(\begin{array}{ccc}
-\frac{a b}{(a+b)} & -z & -y \\
z & a & x \\
y & x & b
\end{array}\right) .
$$

The Lyapunov exponents' calculations for fractionalorder systems were first proposed by Wolf et al. [52] in the context of integer-order derivative. The procedure of the calculation is presented in detail in [52]. In the fractional context, Danca and Kuznetsov [53] have modified the original Matlab algorithm by replacing the part where Ode 45 of the system is used by a numerical scheme of the fractional differential equations (fde12). Furthermore, in the algorithm procedure, the Jacobian matrix is fundamental; for more pieces of information related to the Danca and Kuznetsov algorithms, see [53]. Before continuing, it is essential to recall that many characterizations associated with the Lyapunov exponent sign and the zero into the Lyapunov exponents to characterize periodic behaviors, chaotic behaviors, and hyperchaotic behaviors are not adequate definitions and depend on the values of the fractional-order 
derivative [54]. Therefore, Lyapunov exponents to characterize the nature of chaos in a fractional context are a new open problem [54]. In the first case, we consider the order $\alpha=0.88$, and we establish the Lyapunov exponents. Considering the Jacobian matrix and the numerical scheme, we get the following Lyapunov exponents:

$$
\begin{aligned}
\text { LE1 } & =1.9582, \\
\text { LE2 } & =0.2192, \\
\text { LE3 } & =-20.2852 .
\end{aligned}
$$

It is straightforward to notice that the considered fractional-order system is dissipative because the sum of the Lyapunov exponents is negative. Furthermore, the system admits hyperchaotic behaviors at the order $\alpha=0.88$ because LE1 and LE2 are positive and large. The dimension of the Lyapunov exponent in this context is given by

$$
\operatorname{dim}(\mathrm{LE})=2+\frac{\mathrm{LE} 1+\mathrm{LE} 2}{|\mathrm{LE} 3|}=2.1036
$$

In the second case, we consider the order $\alpha=0.92$, and we establish the Lyapunov exponents. Considering the Jacobian matrix in work, we get the following Lyapunov exponents:

$$
\begin{aligned}
\text { LE1 } & =1.6995, \\
\text { LE2 } & =0.0512, \\
\text { LE3 } & =-16.9046 .
\end{aligned}
$$

It is straightforward to notice that the considered system is dissipative because the sum of the Lyapunov exponents is negative. Furthermore, the system admits high hyperchaotic behaviors at the order $\alpha=0.92$ because there exist two large Lyapunov exponents that are positive. The dimension associated with the Lyapunov exponents in this context is given by

$$
\operatorname{dim}(\mathrm{LE})=2+\frac{\mathrm{LE} 1+\mathrm{LE} 2}{|\mathrm{LE} 3|}=2.1066
$$

In the last case, we consider the order $\alpha=0.95$, and we establish the Lyapunov exponents. Considering the Jacobian matrix and the numerical scheme, we get the following Lyapunov exponents:

$$
\begin{aligned}
& \mathrm{LE} 1=1.7174, \\
& \mathrm{LE} 2=-0.0832, \\
& \mathrm{LE} 3=-15.3319 .
\end{aligned}
$$

It is straightforward to notice that the considered system is dissipative because the sum of the Lyapunov exponents is negative. Furthermore, the system admits high chaotic behaviors at the order $\alpha=0.95$ because there exists one maximal positive and large Lyapunov exponent LE1. The dimension associated with the Lyapunov exponents in this context is given by

$$
\operatorname{dim}(\mathrm{LE})=2+\frac{\mathrm{LE} 1+\mathrm{LE} 2}{|\mathrm{LE} 3|}=2.1066
$$

In conclusion, we can observe various types of chaos when the order $\alpha$ varies. Therefore, fractional-order derivative has a role in generating new types of chaos in general.

The second part of this section is to quantify the chaotic behaviors and hyperchaotic behaviors when the parameters have small changes. Alternatively, we calculate the Lyapunov exponent versus the variations of the different parameters. In our first case, we suppose that the parameter $a$ varies. For simplification, in the rest of the text, we work with the order $\alpha=0.92$. Before calculating the Lyapunov exponents, we represent the phase portraits at the point $a=-15$ to observe the impact of the parameter $a$ in the dynamics. Note that $b$ and $c$ do not change. We depict in Figure 13 the dynamics of the fractional-order system in (13)-(15) in context of $x, y$, and $z$ directions.

We depict in Figure 14 the dynamics of the fractionalorder system in (13)-(15) in context of $y$ and $z$ directions, with the order $\alpha=0.92$.

We depict in Figure 15 the dynamics of the fractionalorder system in (13)-(15) in context of $x$ and $z$ directions, with the order $\alpha=0.92$.

We depict in Figure 16 the dynamics of the fractionalorder system in (13)-(15) in context of $x$ and $y$ directions, with the order $\alpha=0.92$.

Comparing between $a=-10$ and $a=-15$, we can observe that the parameter $a$ has a significant impact on the dynamics of the system in (13)-(15). We confirm the existence of chaotic behaviors by calculating the Lyapunov exponents. In Table 1, the values of the Lyapunov exponents according to the variation of the parameter $a$ in $(-15,-10)$ are mentioned.

We observe that when $a=-15$, we notice by the Lyapunov exponents the existence of high chaotic behaviors because, at this point, there exists one positive Lyapunov exponent LE1 at all time series. The system is chaotic at $a=$ -15 and hyperchaotic at $a=-10$; these different phenomena are due to the fractional-order impact.

Let us see the impact of the parameter $b$; we depict the next figures by considering $b=-2, a=-10$, and $c=0$. We depict in Figure 17 the dynamics of the fractional-order system in (13)-(15) in context of $x, y$, and $z$ directions.

We depict in Figure 18 the dynamics of the fractionalorder system in (13)-(15) in context of $y$ and $z$ directions, with the order $\alpha=0.92$.

We depict in Figure 19 the dynamics of the fractionalorder system in (13)-(15) in context of $x$ and $z$ directions, with the order $\alpha=0.92$.

We depict in Figure 20 the dynamics of the fractionalorder system in (13)-(15) in context of $x$ and $y$ directions, with the order $\alpha=0.92$.

Comparing $b=-2$ and $b=-4$, we can notice the influence of the parameter $b$ in chaotic behaviors. The Lyapunov exponents' values have no sense in this present context because the chaotic behavior is removed.

In this last step, we work with the parameter $c$. By depicting the phase portraits, we can observe that when $c$ varies into $(0,5)$ (approximately), the phase portraits in the section do not have a significant change. Here, we represent the figures with $c=10, a=-10$, and $b=-4$. We depict in 


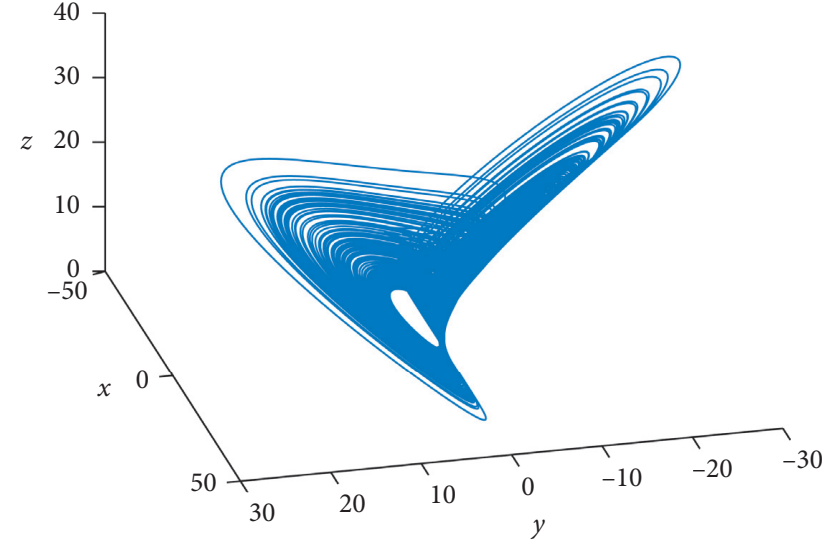

Figure 13: Dynamics of the fractional-order system in (13)-(15) with the order $\alpha=0.92$, in context of $x, y$, and $z$ directions.

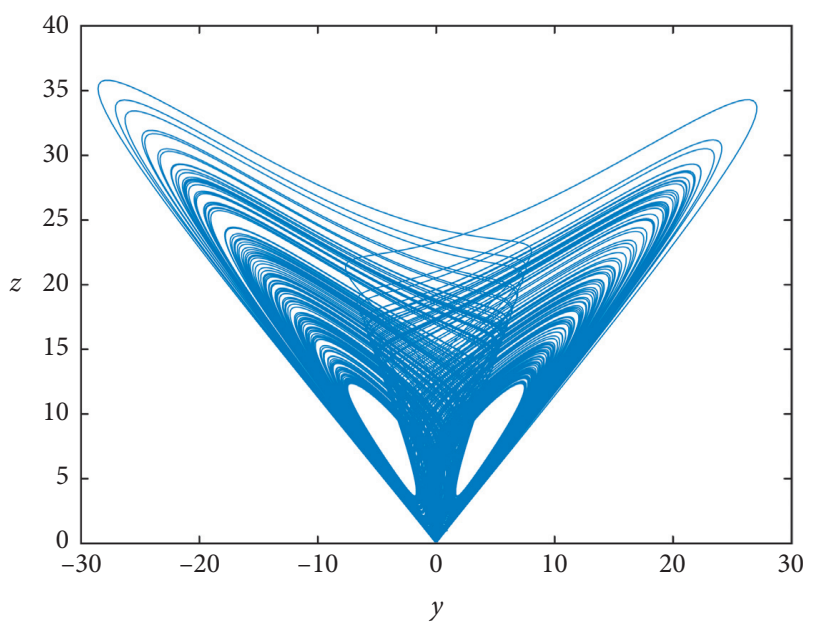

Figure 14: Dynamics of the fractional-order system in (13)-(15) with the order $\alpha=0.92$, in context of $y$ and $z$ directions.

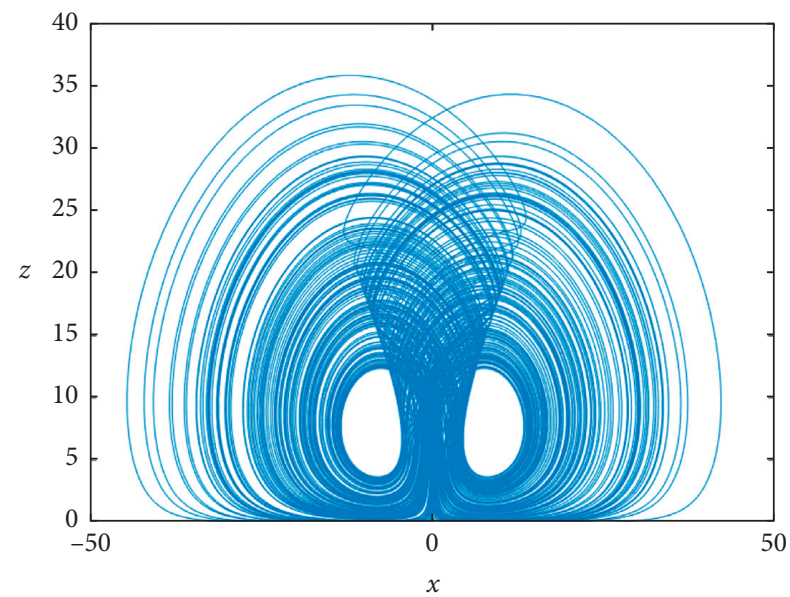

Figure 15: Dynamics of the fractional-order system in (13)-(15) with the order $\alpha=0.92$, in context of $x$, and $z$ directions.

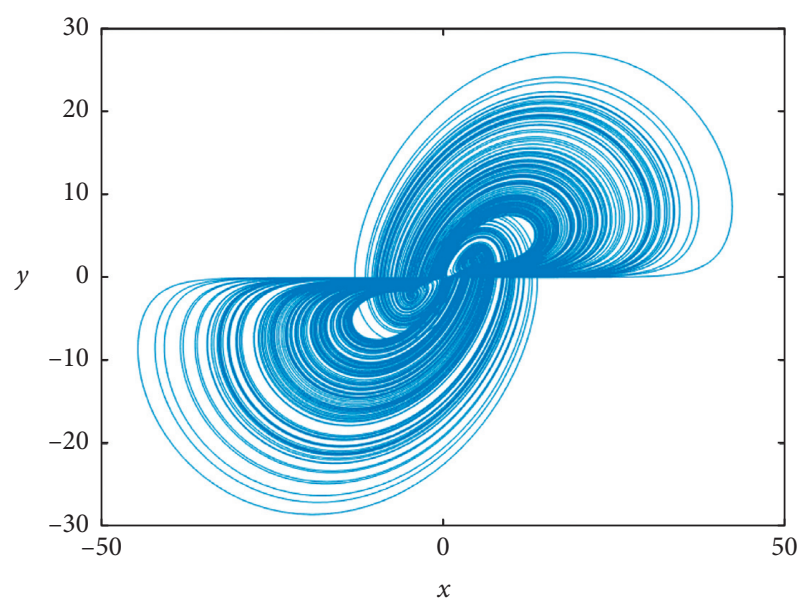

Figure 16: Dynamics of $\mathrm{Lu}$ et al.'s fractional-order system in (13)-(15) with the order $\alpha=0.92$, in context of $x$ and $y$ directions.

TABLE 1: Lyapunov exponents.

\begin{tabular}{lccc}
\hline$a$ & LE1 & LE2 & LE3 \\
\hline-15 & 1.2637 & -0.0167 & -23.1076 \\
-14.5 & 1.2561 & -0.4332 & -22.0595 \\
-14.00 & 1.8478 & -0.9793 & -21.4578 \\
-13.50 & 1.5158 & -0.3005 & -21.1476 \\
-13.00 & 1.3479 & -0.2348 & -20.4082 \\
-12.50 & 1.2601 & -0.1954 & -19.7284 \\
-12.00 & 1.4013 & -0.6018 & -18.8277 \\
-11.50 & 1.3435 & -0.1300 & -18.6001 \\
-11.00 & 1.6356 & 0.0689 & -18.4507 \\
-10.50 & 1.6753 & -0.0777 & -17.7066 \\
-10.00 & 1.6995 & 0.0512 & -16.9046 \\
\hline
\end{tabular}

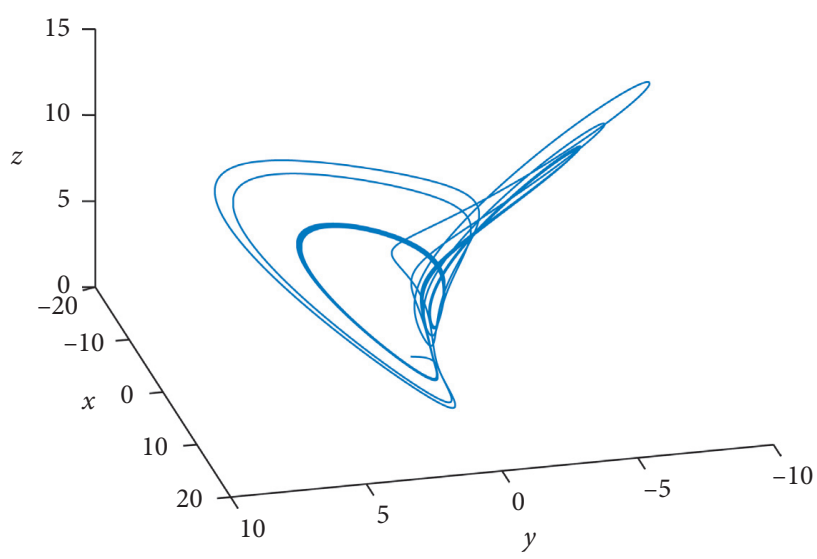

FIgURE 17: Dynamics of the fractional-order system in (13)-(15) with the order $\alpha=0.92$, in context of $x, y$, and $z$ directions.

Figure 21 the dynamics of the fractional-order system in (13)-(15) in the context of $x, y$, and $z$ directions, with the order $\alpha=0.92$.

We depict in Figure 22 the dynamics of the fractionalorder system in (13)-(15) in context of $y$ and $z$ directions, with the order $\alpha=0.92$. 


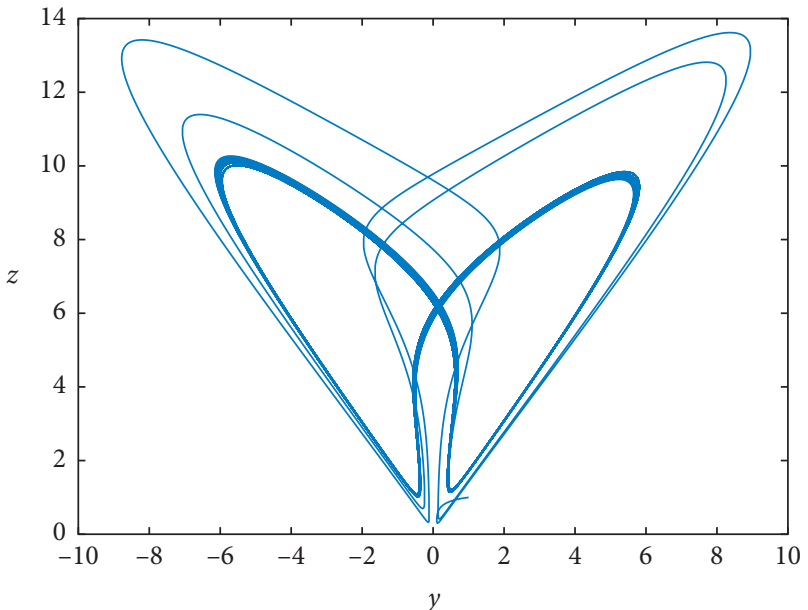

Figure 18: Dynamics of the fractional-order system in (13)-(15) with the order $\alpha=0.92$, in context of $y$ and $z$ directions.

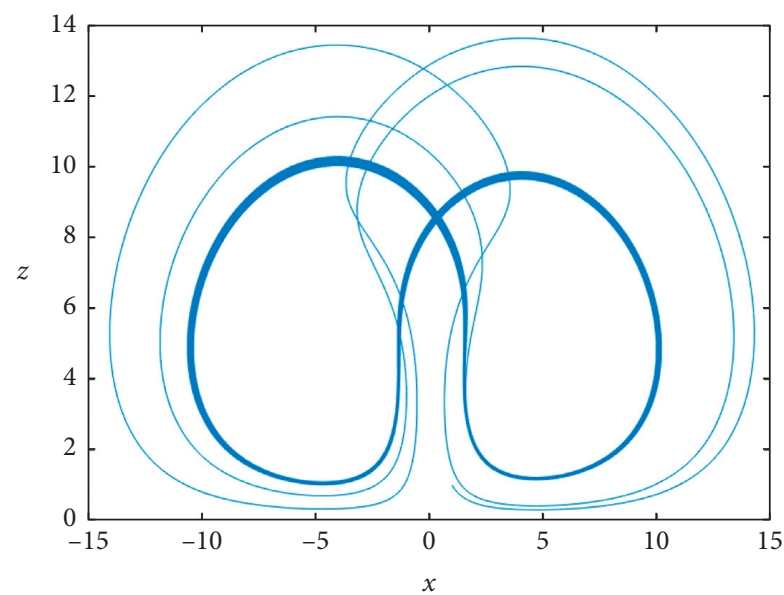

Figure 19: Dynamics of the fractional-order system in (13)-(15) with the order $\alpha=0.92$, in context of $x$ and $z$ directions.

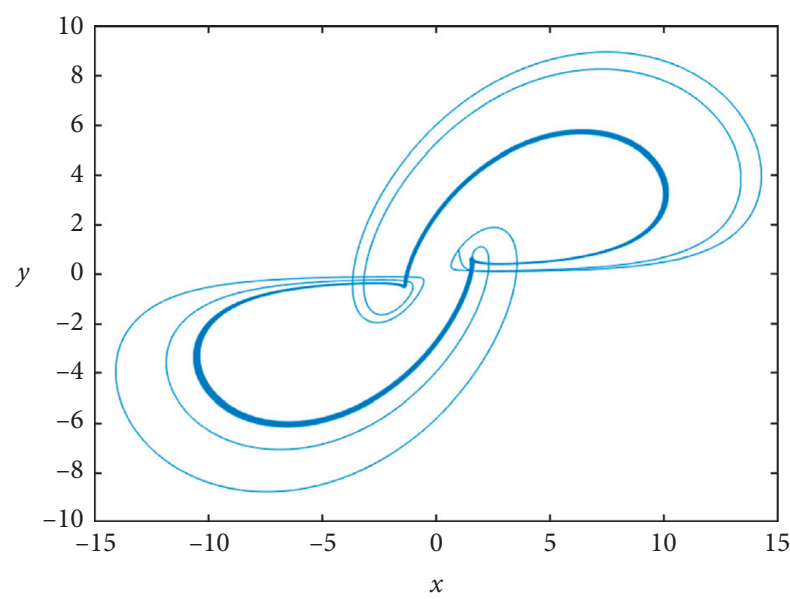

Figure 20: Dynamics of the fractional-order system in (13)-(15) with the order $\alpha=0.92$, in context of $x$ and $y$ directions.

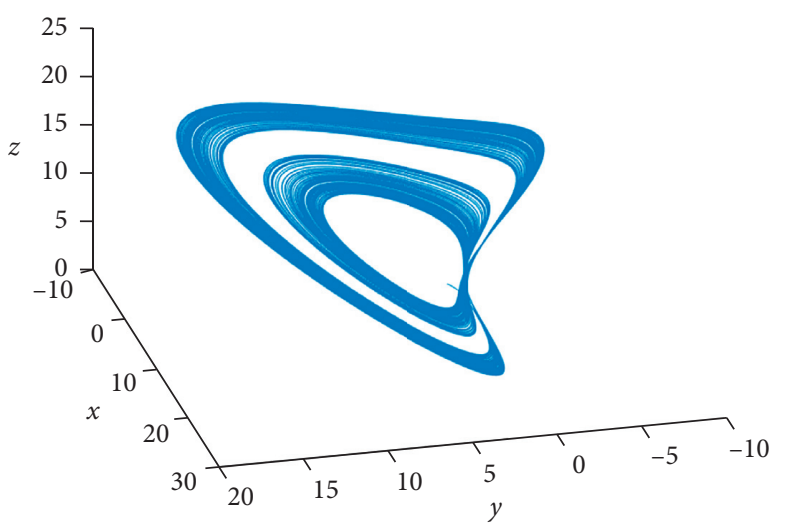

FIgURE 21: Dynamics of the fractional-order system in (13)-(15) with the order $\alpha=0.92$, in context of $x, y$, and $z$ directions.

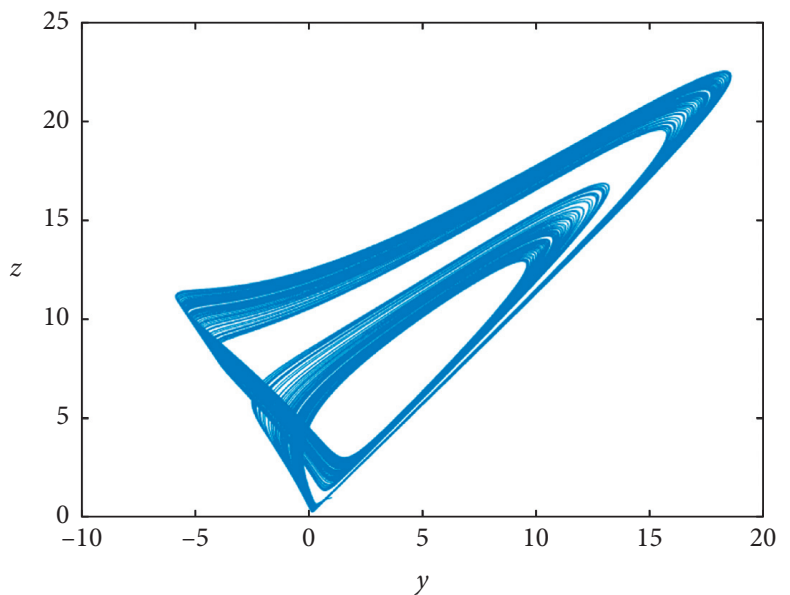

FIgURe 22: Dynamics of the fractional-order system in (13)-(15) with the order $\alpha=0.92$, in context of $y$ and $z$ directions.

We depict in Figure 23 the dynamics of the fractionalorder system in (13)-(15) in context of $x$ and $z$ directions, with the order $\alpha=0.92$.

We depict in Figure 24 the dynamics of the fractionalorder system in (13)-(15) in context of $x$ and $y$ directions, with the order $\alpha=0.92$.

In terms of comparison, we also notice that the parameter $c$ has a significant impact on the considered chaotic system dynamics. For confirmation on the behaviors' changes, we calculate the Lyapunov exponents versus the variation of the parameter $c$. The results are assigned in Table 2.

Table 2 confirms the existence of chaotic behaviors when the parameter $c$ exceeds 5 , as previously mentioned. Note that the presence of one positive Lyapunov exponent justifies chaotic behaviors for our fractional-order system.

\section{Stability Analysis in Fractional Context}

The local stability in the context of the fractional derivative is essential when the new model is under investigation because the values of the fractional-order derivative have a 


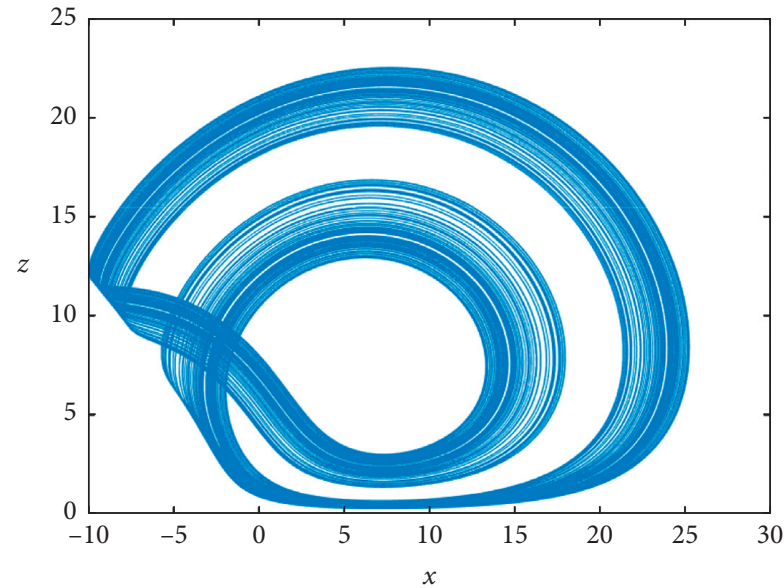

Figure 23: Dynamics of the fractional-order system in (13)-(15) with the order $\alpha=0.92$, in context of $x$ and $z$ directions.

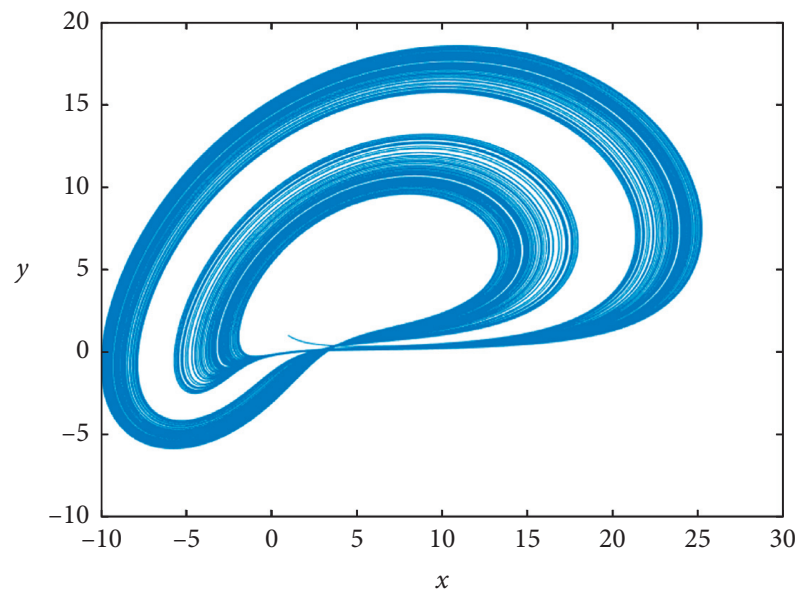

FIgURE 24: Dynamics of the fractional-order system in (13)-(15) with the order $\alpha=0.92$, in context of $x$ and $y$ directions.

TABLE 2: Lyapunov exponents.

\begin{tabular}{lccc}
\hline$c$ & LE1 & LE2 & LE3 \\
\hline 5.00 & 1.3013 & -0.2933 & -16.5138 \\
5.50 & 1.2724 & -0.0990 & -16.6866 \\
6.00 & 1.4717 & -0.1921 & -16.7807 \\
6.50 & 1.6340 & -0.0288 & -17.0811 \\
7.00 & 1.2979 & -0.1671 & -16.6348 \\
7.50 & 0.4083 & -0.3907 & -13.7497 \\
8.00 & 1.3086 & -0.6504 & -16.1780 \\
8.50 & 1.2556 & -0.6855 & -16.0917 \\
9.00 & 1.0302 & -0.6750 & -15.8786 \\
9.50 & 1.1671 & -0.7169 & -15.9639 \\
10.00 & 1.4287 & -0.1244 & -16.8031 \\
\hline
\end{tabular}

significant impact on the dynamics of the fractional-order system in (13)-(15). Therefore, in this section, we will focus on the local stability of the equilibrium points of the fractional-order system described by the Caputo fractionalorder derivative. The question will be how this study will be investigated. Is it the same as the classical method in the context of integer derivative? Is it an algebraic method? The technique employed in the fractional context differs from the method used in the context of integer order. Here we will use the criterion called the Matignon criterion, which is used in the fractional context $[55,56]$. The procedure is to verify whether all the equilibrium points satisfy Matignon criterion given by

$$
|\arg (\lambda(J))|>\frac{\alpha \pi}{2}
$$

where $\lambda(J)$ is the eigenvalue of the Jacobian matrix associated with the equilibrium point under consideration. After resolution of the model described in equations (13)-(15), we have to solve

$$
\begin{aligned}
& 0=-\frac{a b}{a+b} x-y z+c, \\
& 0=a y+x z \\
& 0=b z+x y
\end{aligned}
$$

The equilibrium points in the case of the parameter $c$ being nonnull are already established in the literature. Here we investigate with $c=0$ and we get the following equilibrium points: $P_{1}=(0,0,0), \quad P_{2}=(-6.3245,3.3805$, -5.3450), $\quad P_{3}=(6.3245,3.3805,5.3450), \quad P_{4}=(-6.3245$, $-3.3805,5.3450)$, and $P_{5}=(6.3245,-3.3805,-5,3450)$. We recall that the Jacobian matrix of equations (13)-(15) is given by

$$
J=\left(\begin{array}{ccc}
-\frac{a b}{(a+b)} & -z & -y \\
z & a & x \\
y & x & b
\end{array}\right) .
$$

We firstly evaluate the local stability at the point $P_{1}=(0,0,0)$. Its associated Jacobian matrix is described in the following form:

$$
J=\left(\begin{array}{ccc}
2.8571 & 0 & 0 \\
0 & -10 & 0 \\
0 & 0 & -4
\end{array}\right)
$$

The eigenvalues of the previous matrix are as follows: $\lambda_{1}=2.8571, \lambda_{2}=-10$, and $\lambda_{3}=-4$. We notice that the first eigenvalue satisfies $\left|\arg \left(\lambda_{1}\right)\right|=0<\alpha \pi / 2$, which contradicts the condition represented in equation (47). Thus, the point $P_{1}=(0,0,0)$ is not stable.

We secondly evaluate the local stability at the point $P_{2}=(-6.3245,3.3805,-5.3450)$. Its associated Jacobian matrix is described in the following form:

$$
J=\left(\begin{array}{ccc}
2.8571 & 5.3450 & -3.3805 \\
-5.3450 & -10 & -6.3245 \\
3.3805 & -6.3245 & -4
\end{array}\right) \text {. }
$$


The eigenvalues of the previous matrix are as follows: $\lambda_{1}=1.2338+5.6623 i, \quad \lambda_{2}=1.2338-5.6623 i, \quad$ and $\quad \lambda_{3}=$ -13.6106 . The eigenvalues satisfy the following relationships: $\left|\arg \left(\lambda_{1}\right)\right|=77 \pi / 180>\alpha \pi / 2$ for all $\alpha<0.85,\left|\arg \left(\lambda_{2}\right)\right|=$ $77 \pi / 180>\alpha \pi / 2$ for all $\alpha<(0.85)$, and $\left|\arg \left(\lambda_{3}\right)\right|=\pi>\alpha \pi / 2$ for all $\alpha \in(0,1)$. We conclude that the point $P_{2}=(6.3245$, $3.3805,5.3450)$ is not stable when the order exceeds 0.85 .

We thirdly evaluate the local stability at the point $P_{3}=(6.3245,3.3805,5.3450)$. Its associated Jacobian matrix is described in the following form:

$$
J=\left(\begin{array}{ccc}
2.8571 & -5.3450 & -3.3805 \\
5.3450 & -10 & 6.3245 \\
3.3805 & 6.3245 & -4
\end{array}\right) \text {. }
$$

The eigenvalues of the previous matrix are as follows: $\lambda_{1}=1.2338+5.6623 i, \quad \lambda_{2}=1.2338-5.6623 i, \quad$ and $\lambda_{3}=$ -13.6106 . The eigenvalues satisfy the following relationships: $\left|\arg \left(\lambda_{1}\right)\right|=77 \pi / 180>\alpha \pi / 2$ for all $\alpha<0.85,\left|\arg \left(\lambda_{2}\right)\right|=$ $77 \pi / 180>\alpha \pi / 2$ for all $\alpha<(0.85)$, and $\left|\arg \left(\lambda_{3}\right)\right|=\pi>\alpha \pi / 2$ for all $\alpha \in(0,1)$. We conclude that the point $P_{3}=(6.3245$, $3.3805,5.3450)$ is not stable when the order exceeds 0.85 .

We fourthly evaluate the local stability at the point $P_{4}=(-6.3245,-3.3805,5.3450)$. Its associated Jacobian matrix is described in the following form:

$$
J=\left(\begin{array}{ccc}
2.8571 & -5.3450 & 3.3805 \\
5.3450 & -10 & -6.3245 \\
-3.3805 & -6.3245 & -4
\end{array}\right) .
$$

The eigenvalues of the previous matrix are as follows: $\lambda_{1}=1.2338+5.6623 i, \quad \lambda_{2}=1.2338-5.6623 i, \quad$ and $\lambda_{3}=$ -13.6106 . The eigenvalues satisfy the following relationships: $\left|\arg \left(\lambda_{1}\right)\right|=77 \pi / 180>\alpha \pi / 2$ for all $\alpha<0.85,\left|\arg \left(\lambda_{2}\right)\right|=$ $77 \pi / 180>\alpha \pi / 2$ for all $\alpha<(0.85)$, and $\left|\arg \left(\lambda_{3}\right)\right|=\pi>\alpha \pi / 2$ for all $\alpha \in(0,1)$. We conclude that the point $P_{4}=(-6.3245$, $-3.3805,5.3450)$ is not stable when the order exceeds 0.85 .

We lastly evaluate the local stability at the point $P_{5}=(6.3245,-3.3805,-5,3450)$. Its associated Jacobian matrix is described in the following form:

$$
J=\left(\begin{array}{ccc}
2.8571 & 5.3450 & 3.3805 \\
-5.3450 & -10 & 6.3245 \\
-3.3805 & 6.3245 & -4
\end{array}\right) .
$$

The eigenvalues of the previous matrix are as follows: $\lambda_{1}=1.2338+5.6623 i, \quad \lambda_{2}=1.2338-5.6623 i, \quad$ and $\lambda_{3}=$ -13.6106 . The eigenvalues satisfy the following relationships: $\left|\arg \left(\lambda_{1}\right)\right|=77 \pi / 180>\alpha \pi / 2$ for all $\alpha<0.85,\left|\arg \left(\lambda_{2}\right)\right|=$ $77 \pi / 180>\alpha \pi / 2$ for all $\alpha<(0.85)$, and $\left|\arg \left(\lambda_{3}\right)\right|=\pi>\alpha \pi / 2$ for all $\alpha \in(0,1)$. We conclude that the point $P_{5}=(-6.3245$, $-3.3805,5.3450)$ is not stable when the order exceeds 0.85 .

This section's main conclusion is that all the points are not stable when the Caputo derivative exceeds the order $\alpha=0.85$. We can observe that all the eigenvalues except the trivial equilibrium point prove the symmetry the fractionalorder system considered in this paper under the changes of variables $(x,-y,-z),(-x,-y, z)$, and $(-x, y,-z)$.

\section{Conclusion}

We focused in this paper on the local stability analysis of the fractional-order system's equilibrium points. When the fractional derivative order exceeds 0.85 , all the points of the considered fractional-order system are not stable. Therefore, the fractional-order system respects one of the most important chaos system properties. There is instability of the equilibrium points. The phase portraits represented for the fractional-order system provided the fractional order that have various impacts on the model's dynamics. Another finding is related to the Lyapunov exponents in the fractional context. The calculations of the Lyapunov exponents permit us to detect two types of dynamics: the chaotic behaviors and hyperchaotic behaviors. The Lyapunov exponents' calculations to detect the nature of chaos in the fractional context are of paramount importance in the present investigations. The existence and uniqueness of our considered model's solution have been discussed in terms of the Banach fixedpoint theorem to support the numerical approach used in this paper to depict the possible phase portraits. A question is necessary for future investigation: how can the Lyapunov exponent be calculated to characterize the chaos when the system is incommensurable? In the context of an incommensurable system, how can the stability analysis be investigated? What will be the impact of the parameters and the fractional orders in the context of an incommensurable system? Many directions of investigations are asked in the above questions and are open for future studies.

\section{Data Availability}

No data were used to support this study.

\section{Conflicts of Interest}

The authors declare that they have no conflicts of interest.

\section{References}

[1] D. Kumar and S. Kumar, "Construction of four dimensional chaotic finance model and its applications," International Journal of Pure and Applied Mathematics, vol. 118, no. 22, pp. 1171-1187, 2018.

[2] M. Yavuz, "Characterizations of two different fractional operators without singular kernel," Mathematical Modelling of Natural Phenomena, vol. 14, no. 3, p. 302, 2019.

[3] Z. Hammouch, T. Mekkaoui, and P. Agarwal, "Optical solitons for the calogero-bogoyavlenskii-schiff equation in $(2+1)$ dimensions with time-fractional conformable derivative," European Physical Journal Plus, vol. 133, no. 7, p. 248, 2018.

[4] K. Saad, D. Baleanu, and A. Atangana, "New fractional derivatives applied to the korteweg-de vries and korteweg-de vries-burger's equations," Journal of Computational and Applied Mathematics, vol. 37, no. 6, 2019.

[5] N. Sene and A. N. Fall, "Homotopy perturbation $\rho$-laplace transform method and its application to the fractional diffusion equation and the fractional diffusion-reaction equation," Fractal and Fractional, vol. 3, no. 2, p. 14, 2019. 
[6] N. Sene, "Analytical solutions and numerical schemes of certain generalized fractional diffusion models," European Physical Journal Plus, vol. 134, p. 199, 2019.

[7] N. Sene, "Second-grade fluid model with caputo-liouville generalized fractional derivative," Chaos, Solitons \& Fractals, vol. 133, Article ID 109631, 2020.

[8] N. Sene, "SIR epidemic model with mittag-leffler fractional derivative," Chaos, Solitons \& Fractals, vol. 137, Article ID 109833, 2020.

[9] C. Chen, T. Fan, and B. Wang, "Inverse optimal control of hyperchaotic finance system," World Journal of Modelling and Simulation, vol. 10, no. 2, pp. 83-91, 2014.

[10] D. G. Prakasha and P. Veeresha, "Analysis of Lakes pollution model with mittag-leffler kernel," Journal of Ocean Engineering and Science, 2020.

[11] M. Yavuz and N. Sene, "Stability analysis and numerical computation of the fractional predator-prey model with the harvesting rate," Fractal and Fractional, vol. 4, no. 3, p. 35, 2020.

[12] E. Ahmed, A. M. A. El-Sayed, and H. A. A. El-Saka, “On some routh-hurwitz conditions for fractional order differential equations and their applications in lorenz, rössler, chua and chen systems," Physics Letters A, vol. 358, no. 1, pp. 1-4, 2006.

[13] A. Kumar, H. V. S. Chauhan, C. Ravichandran, K. S. Nisar, and D. Baleanu, "Existence of solutions of non-autonomous fractional differential equations with integral impulse condition," Advances in Difference Equations, vol. 2020, p. 434, 2020.

[14] S. K. Panda, T. Abdeljawad, and C. Ravichandran, "Novel fixed point approach to atangana-baleanu fractional and lpfredholm integral equations," Alexandria Engineering Journal, vol. 59, no. 4, pp. 1959-1970, 2020.

[15] N. Sene, "Global asymptotic stability of the fractional differential equations," Journal of Nonlinear Sciences and Applications, vol. 13, pp. 171-175, 2020.

[16] J. Fahd, T. Abdeljawad, and D. Baleanu, "On the generalized fractional derivatives and their caputo modification," Journal of Nonlinear Sciences and Applications, vol. 10, pp. 2607-2619, 2017.

[17] A. A. Kilbas, H. M. Srivastava, and J. J. Trujillo, Theory and Applications of Fractional Differential Equations, Elsevier, Amsterdam, Netherlands, 2006.

[18] I. Podlubny, Fractional Differential Equations, Mathematics in Science and Engineering, Academic Press, New York, NY, USA, 1999.

[19] A. Atangana and D. Baleanu, "New fractional derivatives with nonlocal and non-singular kernel: theory and application to heat transfer model," Thermal Science, vol. 20, no. 2, pp. 763-769, 2016.

[20] M. Caputo and M. Fabrizio, "A new definition of fractional derivative without singular kernel," Progress in Fractional Differentiation and Applications, vol. 1, no. 2, pp. 1-15, 2015.

[21] A. K. Alomari, "A novel solution for fractional chaotic chen system," Journal of Nonlinear Sciences and Applications, vol. 8, no. 5, pp. 478-488, 2015.

[22] H. Aminikhah, A. Refahi Sheikhani, and H. Rezazadeh, "Stability analysis of distributed order fractional chen system," The Scientific World Journal, vol. 2013, Article ID 645080, 13 pages, 2013.

[23] G. Chen and T. Ueta, "Yet another chaotic attractor," International Journal of Bifurcation and Chaos, vol. 9, no. 7, pp. 1465-1466, 1999.

[24] J. Lü, G. Chen, and D. Cheng, “A new chaotic system and beyond: the generalized lorenz-like system," International
Journal of Bifurcation and Chaos, vol. 14, no. 5, pp. 1507-1537, 2004.

[25] I. Petras, "A note on the fractional-order chua's system," Chaos, Solitons \& Fractals, vol. 38, pp. 140-147, 2008.

[26] C. Zhou, Z. Li, Y. Zeng, and S. Zhang, "A novel 3D fractionalorder chaotic system with multifarious coexisting attractors," International Journal of Bifurcation and Chaos, vol. 29, no. 1, Article ID 1950004, 2019.

[27] M. Diouf and N. Sene, "Analysis of the financial chaotic model with the fractional derivative operator," Complexity, vol. 2020, Article ID 9845031, 14 pages, 2020.

[28] S. Zhang, Y. Zeng, Z. Li, and C. Zhou, "Hidden extreme multistability, antimonotonicity and offset boosting control in a novel fractional-order hyperchaotic system without equilibrium," International Journal of Bifurcation and Chaos, vol. 28, no. 13, Article ID 1850167, 2018.

[29] A. Akgul, I. Moroz, I. Pehlivan, and S. Vaidyanathan, "A new four-scroll chaotic attractor and its engineering applications," Optik, vol. 127, no. 13, pp. 5491-5499, 2016.

[30] Z. Hammouch and T. Mekkaoui, "Control of a new chaotic fractional-order system using mittag-leffler stability," Nonlinear Studies, vol. 22, no. 4, pp. 1-13, 2015.

[31] C. Jiang, S. Liu, and C. Luo, "A new fractional-order chaotic complex system and its antisynchronization," Abstract and Applied Analysis, vol. 2014, Article ID 326354, 12 pages, 2014.

[32] S. Ren, S. Panahi, K. Rajagopal, A. Akgul, V.-T. Pham, and S. Jafari, "A new chaotic flow with hidden attractor: the first hyperjerk system with no equilibrium," Zeitschrift für Naturforschung A, vol. 73, no. 3, pp. 239-249, 2018.

[33] S. Vaidyanathan, A. Akgul, S. Kacar, and U. Cavusoglu, "A new 4-D chaotic hyperjerk system, its synchronization, circuit design and applications in RNG, image encryption and chaosbased steganography," European Physical Journal Plus, vol. 133, p. 46, 2018.

[34] Z. Li, T. Xia, and C. Jiang, "Synchronization of fractionalorder complex chaotic systems based on observers," Entropy, vol. 21 , no. 5, p. 481, 2019.

[35] J. Lü, T. Zhou, G. Chen, and S. Zhang, "Local bifurcations of the chen system," International Journal of Bifurcation and Chaos, vol. 12, no. 10, pp. 2257-2270, 2002.

[36] Z. Wang, X. Huang, and G. Shi, "Analysis of nonlinear dynamics and chaos in a fractional order financial system with time delay," Computers \& Mathematics with Applications, vol. 62, no. 3, pp. 1531-1539, 2011.

[37] S. He, K. Sun, H. Wang, X. Mei, and Y. Sun, "Generalized synchronization of fractional-order hyperchaotic systems and its DSP implementation," Nonlinear Dynamics, vol. 92, no. 1, pp. 85-96, 2018.

[38] M. Shirkavand and M. Pourgholi, "Robust fixed-time synchronization of fractional order chaotic using free chattering nonsingular adaptive fractional sliding mode controller design," Chaos, Solitons \& Fractals, vol. 113, pp. 135-147, 2018.

[39] G. Xu, Y. Shekofteh, A. Akgül, C. Li, and S. Panahi, "A new chaotic system with a self-excited attractor: entropy measurement, signal encryption, and parameter estimation," Entropy, vol. 20, no. 2, p. 86, 2018.

[40] S. He, K. Sun, X. Mei, B. Yan, and S. Xu, "Numerical analysis of a fractional-order chaotic system based on conformable fractional-order derivative," European Physical Journal Plus, vol. 132, p. 36, 2017.

[41] K. Rajagopal, A. Akgul, S. Jafari, A. Karthikeyan, and I. Koyuncu, "Chaotic chameleon: dynamic analyses, circuit implementation, fpga design and fractional-order form with 
basic analyses," Chaos, Solitons \& Fractals, vol. 103, pp. 476-487, 2017.

[42] I. Petras, "Fractional-order chaotic systems," Nonlinear Physical Science, Springer, Berlin, Germany, pp. 103-184, 2011.

[43] V.-T. Pham, S. Vaidyanathan, C. Volos, S. Jafari, and S. T. Kingni, "A no-equilibrium hyperchaotic system with a cubic nonlinear term," Optik, vol. 127, no. 6, pp. 3259-3265, 2016.

[44] Z. Ping, C. Yuan-Ming, and K. Fei, "Synchronization between fractional-order chaotic systems and integer orders chaotic systems (fractional-order chaotic systems)," Chinese Physics B, vol. 19, no. 9, Article ID 090503, 2010.

[45] V.-T. Pham, C. Volos, S. Jafari, and T. Kapitaniak, "Coexistence of hidden chaotic attractors in a novel no-equilibrium system," Nonlinear Dynamics, vol. 87, no. 3, pp. 2001-2010, 2017.

[46] X. Yang, H. Liu, and S. Li, "Synchronization of fractionalorder and integer-order chaotic (hyper-chaotic) systems with different dimensions," Advances in Difference Equations, vol. 2017, p. 344, 2017.

[47] S. K. Panda, T. Abdeljawad, and C. Ravichandran, "A complex valued approach to the solutions of Riemann-Liouville integral, atangana-baleanu integral operator and non-linear telegraph equation via fixed point method," Chaos, Solitons \& Fractals, vol. 130, Article ID 109439, 2020.

[48] A. Granas and J. Dugundji, "Elementary fixed point theorems," in Fixed Point TheorySpringer, New York, NY, USA, 2003.

[49] T. Abdeljawad, E. Karapınar, S. K. Panda, and N. Mlaiki, "Solutions of boundary value problems on extended-branciari b-distance," Journal of Inequalities and Applications, vol. 103, pp. 1-16, 2020.

[50] R. Garrappa, "Numerical solution of fractional differential equations: a survey and a software tutorial," Math, vol. 6, no. 2, p. 16, 2019.

[51] P. Veeresha and D. G. Prakasha, "Solution for fractional generalized zakharov equations with mittag-leffler function," Results in Engineering, vol. 5, Article ID 100085, 2020.

[52] A. Wolf, J. B. Swift, H. L. Swinney, and J. A. Vastano, "Determining lyapunov exponents from a time series," Physica D: Nonlinear Phenomena, vol. 16, no. 3, pp. 285-317, 1985.

[53] M.-F. Danca and N. Kuznetsov, "Matlab code for lyapunov exponents of fractional-order systems," International Journal of Bifurcation and Chaos, vol. 28, no. 5, Article ID 1850067, 2018.

[54] M.-F. Danca, "Lyapunov exponents of a discontinuous 4D hyperchaotic system of integer or fractional order," Entropy, vol. 20, no. 5, p. 337, 2018.

[55] D. Matignon, "Stability results on fractional differential equations to control processing," in Proceedings of the Computational Engineering in Systems Applications, Lille, France, 1996.

[56] N. Sene, "Stability analysis of the generalized fractional differential equations with and without exogenous inputs," Journal of Nonlinear Sciences and Applications, vol. 12, no. 9, pp. 562-572, 2019. 